\title{
INVESTIGATION OF PAHs IN GALACTIC PLANETARY NEBULAE WITH THE AKARI/IRC AND THE SPITZER/IRS
}

\author{
R. Ohsawa ${ }^{1}$, T. Onaka ${ }^{1}$, I. Sakon ${ }^{1}$, T. I. Mori ${ }^{1}$, I. Yamamura ${ }^{2}$, M. Matsuura $^{3}$, \\ H. Kaneda ${ }^{4}$, J. Bernard-Salas ${ }^{5}$, O. Berné ${ }^{6}$, and C. Joblin ${ }^{6}$ \\ ${ }^{1}$ Department of Astronomy, Graduate School of Science, The University of Tokyo, Tokyo, Japan, \\ ${ }^{2}$ Japan Aerospace Exploration Agency, Institute of Space and Astronautical Science, Kanagawa, Japan \\ ${ }^{3}$ Department of Physics \& Astronomy, University College London, London, United Kingdom, \\ ${ }^{4}$ Division of Particle and Astrophysical Sciences, G, Nagoya University, Nagoya, Japan, \\ ${ }^{5}$ Institut d'Astrophysique Spatiale, CNRS/Université Paris-Sud, Orsay, France, \\ ${ }^{6}$ Institut de Recherche en Astrophysique et Planétologie, Université de Toulouse/CNRS, France, \\ E-mail: ohsawa@astron.s.u-tokyo.ac.jp
}

(Received June 30, 2012; Accepted August 09, 2012)

\begin{abstract}
Polycyclic aromatic hydrocarbons (PAHs) in Galactic planetary nebulae (PNe) are investigated by means of the unidentified infrared (UIR) bands. Continuous near- to mid-infrared spectra of PNe are obtained with the AKARI/IRC and the Spitzer/IRS. All 19 PNe in the present study show prominent dust emissions and we investigate the variation in the intensity ratios among the UIR bands. The ionization fraction and the size distribution of PAHs in PNe are derived using the UIR band ratios. We find that the ionization fraction of PAHs in PNe is around 0.0-0.6 and that small PAHs are scarce. The present result indicates a systematic trend of the $3.4 \mu \mathrm{m}$ aliphatic feature to become weak as the PAH ionization fraction increases.
\end{abstract}

Key words: infrared; spectroscopy: ISM; PAHs: star; planetary nebula

\section{INTRODUCTION}

The unidentified infrared (UIR) bands are broad and prominent infrared emissions that are seen in a variety of objects (Peeters et al., 2002; Acke et al., 2004), suggesting that the carriers are common in the Universe. The relative strength of the UIR bands is sensitive to the chemical and physical conditions of the carriers. The most probable candidates are polycyclic aromatic hydrocarbons (PAHs) (Duley \& Williams, 1981). The UIR band ratios can be good diagnostic tools to investigate the PAH properties and the local environments (e.g., Berné et al., 2007; Mori et al., 2012). We investigate the ionization fraction and the size distribution of PAHs in PNe by means of the variation in the UIR band ratios. The $3.4 \mu \mathrm{m}$ emission feature is also investigated as a tracer of aliphatic C-H bonds in PAHs.

\section{OBSERVATION}

We obtained 86 near-infrared $(2-5 \mu \mathrm{m})$ spectra of Galactic PNe with the AKARI/IRC. Among them, 19 PNe whose mid-infrared spectra $(5-14 \mu \mathrm{m})$ are available in the Spitzer Archive and which show significant dust emissions in their spectra are selected. To correct for the slit efficiency, the Spitzer/IRS spectra are scaled to the IRC spectra. The intensities of the UIR bands are estimated through spectral fitting. We fit the spectra with a linear combination of free-free, thermal dust, recombination and forbidden lines, and the UIR band emissions. The UIR bands are assumed as Lorentzian functions. 


\section{RESULTS}

The 6.2 to $11.2 \mu \mathrm{m}$ ratio increases when PAHs are ionized, while the 3.3 to $11.2 \mu \mathrm{m}$ ratio increases when small PAHs are abundant (Allamandola et al., 1989). Using the upper panel of Fig. 1, we can diagnose the ionization status and the size distribution of PAHs. Most PNe show a 6.2 to $11.2 \mu \mathrm{m}$ ratio of around $0.5-1.2$. For some PNe, this ratio becomes larger than 2 . The latter also show the eminent broad feature around $12 \mu \mathrm{m}$, which is typically seen in the proto-PNe phase and attributed to SiC. They are significantly young PNe, suggesting that PAHs in those objects are pristine. The $3.3 \mu \mathrm{m}$ band arises from aromatic $\mathrm{C}-\mathrm{H}$ bonds, while the $3.4 \mu \mathrm{m}$ band arises from aliphatic $\mathrm{C}-\mathrm{H}$ bonds. The 3.4 to $3.3 \mu \mathrm{m}$ ratio indicates the aliphatic-to-aromatic ratio. This ratio is shown against the 6.2 to $11.2 \mu \mathrm{m}$ band ratio in the lower panel of Fig. 1, where the young objects with the 6.2 to $11.2 \mu \mathrm{m}$ larger than 2 are excluded.

\section{DISCUSSION}

We calculate the UIR band ratios with different effective temperatures $(T)$ and ionization fractions $\left(f_{\text {ion }}\right)$ based on a PAH model for molecular clouds and photodissociation regions (Case I in Mori et al., 2012) to investigate the difference between the PAHs in PNe and in interstellar fields. The results of the models are shown by the gray lines in the upper panel. Although the 6.2 to $11.2 \mu \mathrm{m}$ band ratio $(0.5-1.2)$ is well reproduced by the model with $f_{\text {ion }} \simeq 0.0-0.5$, the 3.3 to $11.2 \mu \mathrm{m}$ ratio is significantly lower than the model prediction. It suggests that small PAHs are scarce in PNe. In the lower panel, we find that the aliphaticaromatic ratio decreases as the ionization fraction increases. This result confirms that aliphatic $\mathrm{C}-\mathrm{H}$ bonds are fragile to UV-processing. The present result indicates that the destruction of aliphatic bonds occurs as PAHs become ionized in PNe.

\section{ACKNOWLEDGEMENTS}

This research is in part based on observations with AKARI, a JAXA project with the participation of ESA and on archival data obtained with the Spitzer Space Telescope, which is operated by the Jet Propulsion Laboratory, California Institute of Technology under a contract with NASA. It is supported in part by a Grantin-Aid for Scientific Research (23244021) by the Japan Society of Promotion of Science (JSPS).
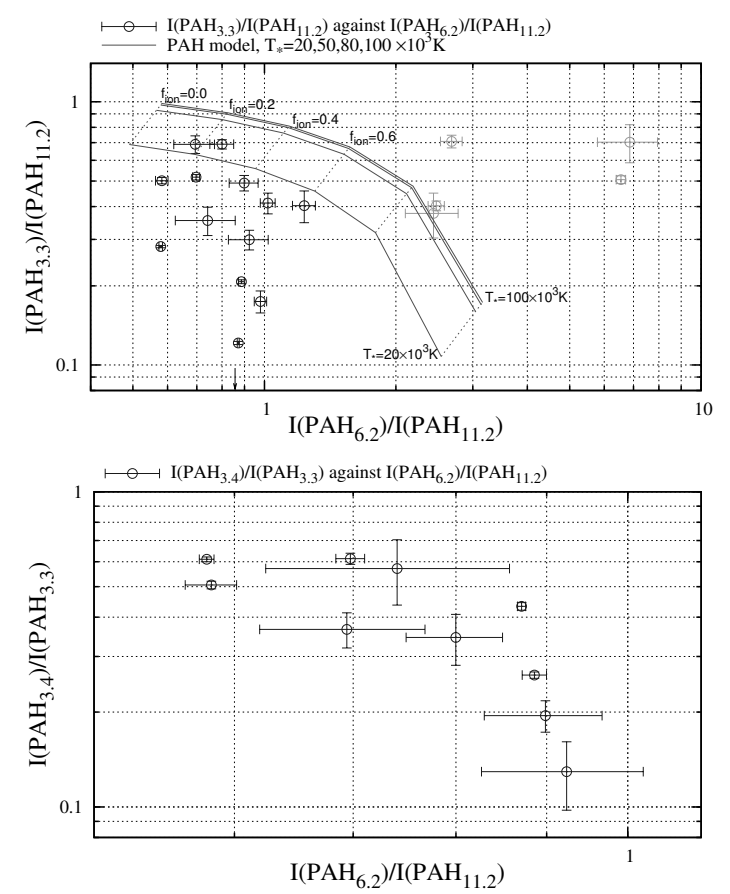

Fig. 1. (Upper) The 3.3 to $11.2 \mu \mathrm{m}$ UIR band ratio is plotted against the 6.2 to $11.2 \mu \mathrm{m}$ ratio. The gray symbols show the objects with the $\mathrm{SiC}$ feature (see text). The solid lines show the band ratio calculated by a PAH model. (Lower) The 3.4 to $3.3 \mu \mathrm{m}$ band ratio is shown against the 6.2 to $11.2 \mu \mathrm{m}$ UIR band ratio.

\section{REFERENCES}

Acke, B. \& van den Ancker, M. E., 2004, ISO Spectroscopy of Disks around Herbig Ae/Be Stars, A\& A, 426, 151

Allamandola, L. J., Tielens, A. G. G. M., \& Barker, J. R., 1989, Interstellar Polycyclic Aromatic Hydrocarbons - The Infrared Emission Bands, The Excitation/Emission Mechanism, and the Astrophysical Implications, ApJS, 71, 733

Berné, O., et al., 2007, Analysis of the Emission of Very Small Dust Particles from Spitzer SpectroImagery Data Using Blind Signal Separation Methods, A\&A, 469, 575

Duley, W. W. \& Williams, D. A., 1981, The Infrared Spectrum of Interstellar Dust - Surface Functional Groups on Carbon, MNRAS, 196, 269

Mori, T. I., et al., 2012, Observations of the Nearto Mid-Infrared Unidentified Emission Bands in the Interstellar Medium of the Large Magellanic Cloud, ApJ, 744, 68

Peeters, E., et al., 2002, The Rich 6 to 9 vec mu m Spectrum of Interstellar PAHs, A\&A, 390, 1089 